\title{
RNA interference-mediated downregulation of phospholipid scramblase 1 expression in primary liver cancer in vitro
}

\author{
LIANG GUI $^{1 *}$, YING-WEI ZHU ${ }^{2 *}$, QIANG XU ${ }^{3 *}$, JU-JU HUANG ${ }^{2}$, PING HUA $^{2}$, \\ GAO-JUE WU ${ }^{2}$, JIAN LU ${ }^{2}$, JING-BIN NI ${ }^{2}$, HONG TANG $^{4}$ and LI-LI ZHANG ${ }^{2}$ \\ ${ }^{1}$ Department of Vascular Surgery, Beijing Hospital, National Center of Gerontology; Institute of Geriatric Medicine, \\ Chinese Academy of Medical Science, Beijing 100730; ${ }^{2}$ Department of Gastroenterology, \\ The Affiliated Wuxi No. 2 People's Hospital of Nanjing Medical University, Wuxi, Jiangsu 214002; \\ ${ }^{3}$ Department of Intervention, Wujin Hospital Affiliated to Jiangsu University, Changzhou, Jiangsu 213002; \\ ${ }^{4}$ Department of Pathology, The Affiliated Wuxi No. 2 People's Hospital of Nanjing Medical University, \\ Wuxi, Jiangsu 214002, P.R. China
}

Received February 10, 2020; Accepted August 5, 2020

DOI: $10.3892 / \mathrm{ol} .2020 .12225$

\begin{abstract}
Phospholipid scramblase 1 (PLSCR1) serves a function in the pathogenesis and progression of various types of cancer. However, the role of PLSCR1 in human primary liver cancer remains unknown. The aim of the present study was to evaluate the expression of PLSCR1 in primary liver cancer and analyse the clinical significance. In addition, the present study detected and compared the biological behaviours of HepG2 cells with different levels of activated PLSCR1 or silenced PLSCR1. PLSCR1 expression in primary liver cancer tissue samples was examined using immunohistochemistry. Cultured HepG2 cells were infected with lentiviruses to suppress or activate PLSCR1 expression. Reverse transcription-quantitative PCR and western blotting were performed to analyse the effects of silencing or activating PLSCR1 in cell lines at the mRNA and protein levels, respectively. The effects of PLSCR1 expression on cell proliferation, adhesion, migration and invasion were subsequently determined using Cell Counting Kit 8, adhesion, and Transwell migration and invasion assays. PLSCR1 expression in primary liver cancer tissue samples was higher compared with that in adjacent non-cancerous liver tissue
\end{abstract}

Correspondence to: Dr Hong Tang, Department of Pathology, The Affiliated Wuxi No. 2 People's Hospital of Nanjing Medical University, 68 Zhongshan Road, Wuxi, Jiangsu 214002, P.R. China E-mail: 843014266@qq.com

Dr Li-Li Zhang, Department of Gastroenterology, The Affiliated Wuxi No. 2 People's Hospital of Nanjing Medical University, 68 Zhongshan Road, Wuxi, Jiangsu 214002, P.R. China E-mail: g118014225672@163.com

*Contributed equally

Key words: phospholipid scramblase 1, human hepatocellular carcinoma, small interfering RNA, lentivirus infection samples and normal tissue samples, and positively correlated with the clinical stage. PLSCR1 was effectively downregulated or overexpressed in HepG2 cells using small interfering RNA and lentivirus techniques, respectively. PLSCR1 upregulation promoted cell proliferation, invasion and migration, while PLSCR1 downregulation inhibited these effects. PLSCR1 is highly expressed in primary liver cancer and associated with the clinical stage. Downregulating the expression of PLSCR1 significantly inhibited the proliferation, adhesion, migration and invasion of cancer cells, suggesting that PLSCR1 may be a potential therapeutic target for preventing the progression of primary liver cancer.

\section{Introduction}

Primary liver cancer is a common type of cancer worldwide with high morbidity and mortality (1). In China, primary liver cancer is the second leading cause of cancer-related death, and the incidence and mortality rates have continued to rise over the decades (2). Primary liver cancer mainly occurs in the context of long-term chronic liver disease, particularly in hepatitis B and C virus infections and cirrhosis (3). Primary liver cancer is characterized as an asymptomatic disease in early stages and is often detected when at advanced stages. Due to being diagnosed in the advanced stages, primary liver cancer is often incurable (4). With the development of medical science multiple advanced treatments, such as radiofrequency ablation, immunotherapy and molecular-targeted therapy have made significant progress in clinical practice (5). However, new methods for early diagnosis and for therapies are urgently required.

Phospholipid scramblase 1 (PLSCR1) is a $\mathrm{Ca}^{2+}$-binding type II endofacial plasma membrane protein that serves an important role in the transbilayer movement of phosphatidylserine and other amino phospholipids (6). These movements are often associated with critical cellular processes, including cell activation, cell proliferation, cell injury responses, tumour suppression, transcriptional regulation and apoptosis (7-12). 
The expression of PLSCR 1 can be regulated by interferons, epidermal growth factor (EGF) and other cytokines (13). Increasing evidence suggests that PLSCR1 also interacts with several protein kinases that contribute to cell signalling pathways, particularly the EGF receptor (EGFR) signalling pathway $(13,14)$. These studies suggest that PLSCR1 may serve an important role in tumorigenesis. PLSCR 1 has been identified to be highly expressed in colorectal carcinoma and breast carcinoma, suggesting that PLSCR1 is closely correlated with tumour initiation and progression (15-17). However, to the best of our knowledge there are no previous studies on the biological functions and expression levels of PLSCR1 in primary liver cancer.

The present study investigated the expression of PLSCR 1 in patients with primary liver cancer and compared biological behaviours among HepG2 cells with different expression levels of PLSCR1. The results of the present study may clarify the association between PLSCR1 and human primary liver cancer.

\section{Materials and methods}

Patient information and tissue samples. A total of 44 patients (male $=31$, female $=11$; age range, $30-80$ years) diagnosed with primary liver cancer at of Wuxi No. 2 People's Hospital between January 2010 and December 2014 were included in the present study. The inclusion criteria were as follows: i) Diagnosed to be primary liver cancer treatable with surgical resection; and ii) all the patients had complete information for clinicopathological and prognostic characteristics. Tumour tissue samples and adjacent noncancerous tissue samples were obtained during surgery and normal tissue samples were obtained from 10 normal individuals recruited (male $=6$, female $=4$; age range, 45-77 years) from the same hospital within the same time frame. Normal individuals were defined as patients with cholelithiasis requiring surgical treatment and preoperative laboratory tests, their imaging examination did not reveal any hepatic lesions. None of the patients received radiotherapy, chemotherapy or other related anti-tumour therapies prior to surgery. Clinicopathological features, including tumour size, differentiation grade and clinical stage, were collected from pathological reports and checked independently by two observers and finally incorporated in Tables I and II. The present study was approved by the Ethics Committee of Wuxi No. 2 People's Hospital (approval no. 2009-Y-22) and written informed consent was obtained from all the patients prior to surgery and from the normal individuals.

Immunohistochemistry and staining assessment. Immunohistochemical staining was performed on serial $4-\mu \mathrm{m}$ tissue sections from formalin-fixed (10\% formalin for $24 \mathrm{~h}$ ), paraffin-embedded human primary liver cancer and adjacent normal samples. Dehydration was performed using a descending alcohol series (70,95 and 100\% ethanol). Firstly, paraffin sections were baked in an oven at $60^{\circ} \mathrm{C}$ for $1 \mathrm{~h}$. The paraffin slides were deparaffinized and washed three times with PBS. The sections were subsequently immersed in $0.01 \mathrm{mmol} / 1 \mathrm{EDTA}$, microwaved at $98^{\circ} \mathrm{C}$ for $25 \mathrm{~min}$ for antigen retrieval and allowed to cool to room temperature (RT). Each section was then washed with PBS, followed by an incubation for $10 \mathrm{~min}$ with $0.3 \% \mathrm{H}_{2} \mathrm{O}_{2}$ to inhibit endogenous peroxidase activity. Each section was then washed with PBS and incubated with $20 \%$ goat serum (Thermo Fisher Scientific, Inc.) for $30 \mathrm{~min}$ at room temperature and a diluted rabbit anti-PLSCR1 polyclonal antibody (1:1,000; cat. no. ab250216, Abcam) for $2 \mathrm{~h}$ at $37^{\circ} \mathrm{C}$ and $2 \mathrm{~h}$ at RT, successively. After being washed with PBS, the slides were incubated with horseradish peroxidase-labeled anti-rabbit IgG secondary antibody (goat, 1:2,000; cat. no. A-1178, Thermo Fisher Scientific, Inc.) for $30 \mathrm{~min}$ at RT. Subsequently, the slides were washed with PBS, incubated with EnVision (Dako; Agilent Technologies, Inc.) for $30 \mathrm{~min}$ at RT and then thoroughly washed with PBS. Subsequently, the sections were developed with diaminobenzidine tetrahydrochloride, washed in running water and counterstained with haematoxylin for $2 \mathrm{~min}$ at RT. Representative samples were selected and images captured. Finally, the sections were observed under an Olympus light microscope (BX61; Olympus Corporation; magnification, $\mathrm{x} 400$ ). Five random fields were selected and evaluated by two blinded experienced pathologists from Wuxi No. 2 People's Hospital (Wuxi, China). The following definition of immunostaining intensity from Kuo et al (17) was used: Negative, no brown particles in the plasma membrane; positive, light-brown particles, moderately brown particles or deep brown particles in the plasma membrane.

Cell lines and cell culture. HepG2 cell lines (purchased from the Cell Bank of the Chinese Academy of Sciences) were cultured in DMEM (Gibco; Thermo Fisher Scientific, Inc.) supplemented with $10 \%$ FBS (Gibco; Thermo Fisher Scientific, Inc.). All cells were maintained at $37^{\circ} \mathrm{C}$ in a humidified incubator with $5 \% \mathrm{CO}_{2}$. STR profiling was performed to ensure the cell identity.

Establishment of cell lines with stable PLSCR1 up- or downregulation. HepG2 cells were seeded in 6-well plates $24 \mathrm{~h}$ prior to viral infection at a density of $2 \times 10^{5}$ cells per well and incubated at $37^{\circ} \mathrm{C}$ with $5 \% \mathrm{CO}_{2}$ overnight. The cells were transiently transfected with synthesized PLSCR1-specific short hairpin (sh)RNAs (sh-PLSCR1, Applied Biological Materials, Inc.), a PLSCR1-expressing lentivirus (lv-PLSCR1, Applied Biological Materials, Inc.) or the corresponding negative control (shnon or Lvnon, respectively, from Applied Biological Materials, Inc.) using Lipofectamine $2000^{\circledR}$ (Invitrogen; Thermo Fisher Scientific, Inc.) after removing the growth medium. Subsequently, $1 \mu$ l of polybrene $(5 \mu \mathrm{g} / \mathrm{ml})$ was added to each well. The medium was changed $24 \mathrm{~h}$ later and replaced with $2 \mathrm{ml}$ of complete medium, and the incubation was continued. Stable PLSCR1-expressing cell lines were harvested following transfection. Reverse transcription-quantitative (RT-q) PCR and western blotting were performed to investigate the expression levels of PLSCR1. Three days later, well-growing monoclonal strains were selected for frozen storage and subsequent experiments.

$R T-q P C R$. Transfected cancer cells were processed with TRIzol ${ }^{\circledR}$ (Life Technologies; Thermo Fisher Scientific, Inc.). Total RNA was reverse transcribed into cDNA using the PrimeScript ${ }^{\mathrm{TM}}$ RT reagent kit (Takara Bio, Inc.) at $50^{\circ} \mathrm{C}$ for $45 \mathrm{~min}$. All the experiments were performed following manufacturer's protocols. qPCR was subsequently performed using the SYBR Green I 
Table I. Protein levels of PLSCR1 in primary liver cancer tissue samples, adjacent non-cancerous liver tissue samples and normal liver tissue samples.

PLSCR1 expression

\begin{tabular}{|c|c|c|c|c|}
\hline \multirow[b]{2}{*}{ Variable } & \multirow[b]{2}{*}{ Cases, $\mathrm{n}$} & & \multirow[b]{2}{*}{ P-value } \\
\hline & & Negative, n (\%) & Positive, n (\%) & \\
\hline Primary liver cancer & 44 & $5(11.36)$ & $39(88.64)$ & $0.082^{\mathrm{a}}$ \\
\hline Adjacent non-cancerous & 44 & $29(65.91)$ & $15(34.09)$ & \\
\hline Normal & 10 & $9(90)$ & $1(10)$ & $<0.001^{\mathrm{b}}$ \\
\hline
\end{tabular}

The estimated P-values were adjusted using Bonferroni corrections. ${ }^{a}$ Primary liver cancer vs. Adjacent non-cancerous; ${ }^{\mathrm{b}}$ primary liver cancer vs. normal. PSCR1, phospholipid scramblase 1.

Table II. Associations between PLSCR1 expression and clinicopathological features in 44 primary liver cancer patients.

\begin{tabular}{|c|c|c|c|c|}
\hline \multirow[b]{2}{*}{ Variable } & \multirow[b]{2}{*}{ Cases, $\mathrm{n}$} & \multicolumn{2}{|c|}{ PLSCR 1 expression } & \multirow[b]{2}{*}{ P-value } \\
\hline & & Negative, n (\%) & Positive, n (\%) & \\
\hline \multicolumn{5}{|l|}{ Age, years } \\
\hline$<60$ & 34 & $3(8.82)$ & $31(91.18)$ & 0.328 \\
\hline$\geq 60$ & 10 & $2(20)$ & $8(80)$ & \\
\hline \multicolumn{5}{|l|}{ Sex } \\
\hline Male & 33 & $3(9.09)$ & $30(90.91)$ & 0.411 \\
\hline Female & 11 & $2(18.18)$ & $9(81.82)$ & \\
\hline \multicolumn{5}{|l|}{ Tumour size, $\mathrm{cm}$} \\
\hline$\leq 3$ & 12 & $2(16.67)$ & $10(83.33)$ & 0.497 \\
\hline$>3$ & 32 & $3(9.38)$ & $29(90.62)$ & \\
\hline \multicolumn{5}{|l|}{ Differentiation } \\
\hline High-moderate & 41 & $5(12.20)$ & $36(87.80)$ & 0.521 \\
\hline Poor & 3 & $0(0)$ & $3(100)$ & \\
\hline \multicolumn{5}{|l|}{ HBs-Ag } \\
\hline Positive & 35 & $1(2.86)$ & $34(97.14)$ & 0.004 \\
\hline Negative & 9 & $4(44.44)$ & $5(55.56)$ & \\
\hline \multicolumn{5}{|l|}{$\mathrm{AFP}, \mu \mathrm{g} / 1$} \\
\hline$>400$ & 20 & $2(10)$ & $18(90)$ & 0.795 \\
\hline$\leq 400$ & 24 & $3(12.5)$ & $21(87.5)$ & \\
\hline \multicolumn{5}{|c|}{ Lymphatic metastasis } \\
\hline Positive & 11 & $0(0)$ & $11(100)$ & 0.411 \\
\hline Negative & 33 & $5(15.15)$ & $28(84.85)$ & \\
\hline \multicolumn{5}{|l|}{ TNM stage } \\
\hline I & 3 & $2(66.67)$ & $1(33.33)$ & 0.045 \\
\hline II & 19 & $2(10.53)$ & $17(89.47)$ & \\
\hline III & 21 & $1(4.76)$ & $20(95.24)$ & \\
\hline IV & 1 & $0(0)$ & $1(100)$ & \\
\hline
\end{tabular}

PSCR1, phospholipid scramblase 1; AFP, $\alpha$ fetoprotein; TNM, tumour node metastasis; HBs-Ag, hepatitis B surface antigen.

Master mix kit (Invitrogen; Thermo Fisher Scientific, Inc.) and a 7500 Real-Time PCR System (Applied Biosystems; Thermo Fisher Scientific, Inc.). The PCR thermocycling conditions were: $95^{\circ} \mathrm{C}$ for $2 \mathrm{~min}$; and 40 cycles of $95^{\circ} \mathrm{C}$ for $10 \mathrm{sec}, 60^{\circ} \mathrm{C}$ for
$30 \mathrm{sec}$ and $72^{\circ} \mathrm{C}$ for $30 \mathrm{sec}$. The sequences of the PCR primers were: PLSCR1-Forward, 5'-CACCCATGTCTACCAAAGTT-3' and PLSCR1-Reverse, 3'-CTCTCAAAATTCCAGTCCAG-5'; $\beta$-actin forward, 5'-GAAGATCAAGATCATTGCTCCT-3' 
and $\beta$-actin reverse, 5'-TACTCCTGCTTGCTGATCCA-3'. The relative gene expression levels were calculated using the $2^{-\Delta \Delta C q}$ method (18) and normalized to the internal reference gene $\beta$-actin. Each sample was run in triplicate and independently repeated three times.

Western blotting. Total protein was extracted from transfected cancer cells using lysis buffer (Beyotime Institute of Biotechnology), and protein concentrations were measured with a Bicinchoninic Acid Protein Assay kit (Beyotime Institute of Biotechnology). A total of $30 \mu 1$ protein extract was separated using $10 \%$ SDS-PAGE gels and transferred onto a PVDF membrane. The membranes were blocked with $5 \%$ non-fat milk for $60 \mathrm{~min}$ at RT, followed by incubation with primary antibodies against PLSCR1 (1:500; cat. no. ab250216; Abcam), EGFR (1:500; cat. no. ab52894; Abcam), and $\beta$-actin (1:500; cat. no. ab8227; Abcam) overnight at $4^{\circ} \mathrm{C}$, then treated with a Horseradish peroxidase-labeled goat anti-rabbit secondary antibody (1:1,000; cat. no. ab150077; Abcam) for $2 \mathrm{~h}$ at RT. The protein bands were visualized using an enhanced chemiluminescence system (Beyotime Institute of Biotechnology). $\beta$-actin was used as a loading control for normalization and images were analyzed using ImageJ software version 1.52 (National Institutes of Health).

CCK 8 assay. Transfected cancer cells $\left(8 \times 10^{3}\right.$ per well) were seeded in 96-well plates in quintuplicate and incubated at $37^{\circ} \mathrm{C}$. DMEM was removed $24 \mathrm{~h}$ later and replaced with $100 \mu \mathrm{l}$ of fresh medium. A Cell Counting Kit-8 (CCK8; Dojindo Molecular Technologies Inc.) assay was used to evaluate cell proliferation according to the manufacturer's instructions. A CCK8 solution $(10 \mu \mathrm{l})$ was added to each well, and the cells were incubated for $4 \mathrm{~h}$ at $37^{\circ} \mathrm{C}$ in a $\mathrm{CO}_{2}$ incubator. The absorbance at $450 \mathrm{~nm}$ was determined by an ELISA plate reader.

Wound heal assay. Cells (3x105/well) were seeded and grown in 6-well plates until confluent (90\%) cell monolayers were formed. Then, a sterile 10- $\mu 1$ pipette tip was used to make a uniform scratch along the bottom of each well. The horizontal distance between the sides of the scratch was measured. The cells were washed with PBS three times to remove detached cells and incubated with serum-free medium at $37^{\circ} \mathrm{C}$ for $24 \mathrm{~h}$ after replacement of the culture medium. Then, wound size was observed and images were captured under an inverted fluorescence microscope (Nikon Corporation; magnification: $\mathrm{x} 20$ ).

Transwell migration and invasion assays. Transwell chambers were used for transfected cancer cell migration and invasion assays. The 24-well culture inserts (BD Biosciences) with $8-\mu \mathrm{m}$ pores coated with Matrigel $(1 \mathrm{mg} / \mathrm{ml}$; BD Biosciences) were used. The membranes of upper chamber were coated with Matrigel (BD) and then incubated for $6 \mathrm{~h}$ at $37^{\circ} \mathrm{C}$. For the migration assay, $2 \times 10^{5}$ transfected cells suspended in $200 \mu \mathrm{l}$ of serum-free medium were seeded in the upper chambers. Medium containing 20\% FBS was added to the lower chamber. For the invasion assay, a Chemicon Cell Invasion Assay kit (Chemicon International; Thermo Fisher Scientific, Inc.) was used. The non-migrating/invading cells were removed from the upper surface of the membrane with cotton swabs following an incubation for $24 \mathrm{~h}$ at $37^{\circ} \mathrm{C}$, and the migrating/invading cells were stained with $0.1 \%$ crystal violet for $30 \mathrm{~min}$ at $\mathrm{RT}$ and images captured with an inverted fluorescence microscope (magnification: x20; Nikon Corporation).

Adhesion assays. A 96-well plate was coated with laminin (Ln, $20 \mu \mathrm{g} / \mathrm{well}$ ) or fibronectin (Fn, $10 \mu \mathrm{g} /$ well) for $2 \mathrm{~h}$ at $37^{\circ} \mathrm{C}$, washed with PBS twice and sealed with $1 \%$ BSA (Thermo Fisher Scientific, Inc.). A single-cell suspension $\left(3 \times 10^{5}\right.$ cancer cells $/ \mathrm{ml}$ ) was prepared with transfected cells and serum-free medium. Then, $200 \mu \mathrm{l}$ of suspension was added to the coated plates. The cells were incubated at $37^{\circ} \mathrm{C}$ with $5 \% \mathrm{CO}_{2}$ for $2 \mathrm{~h}$ and then the non-adherent cells were gently washed off. Each well was fixed with $4 \%$ formaldehyde for $10 \mathrm{~min}$ at RT and stained with $0.5 \%$ hexamethylpararosaniline for $20 \mathrm{~min}$ at RT. Subsequently, $100 \mu \mathrm{l}$ of $10 \%$ SDS was added to each well. An ELISA plate reader was used to assess the absorbance at $570 \mathrm{~nm}$.

Statistical analysis. SPSS 18.0 software (SPSS, Inc.) was used to perform all statistical analyses. Continuous variables are presented as the mean $\pm \mathrm{SD}$ and comparisons between groups were performed using one-way ANOVAs followed by post-hoc Tukey's tests. Categorical data are expressed as a proportion (\%) and were compared using the $\chi^{2}$ test, McNemar's test or Fisher's exact test. Bonferroni's corrections were used to reduce type I errors as the primary liver cancer data in Table I had been analysed more than once. $\mathrm{P}<0.05$ was considered to indicate a statistically significant difference.

\section{Results}

Expression of PLSCR1 in primary liver cancer tissue samples and the associations of PLSCRI levels with clinicopathological variables. Fig. 1 and Table I show that PLSCR1 protein was detected in liver tissue samples through immunohistochemical staining. The PLSCR1 protein was mainly located at the cancer cell membrane (Fig. 1). The expression of PLSCR1 in the primary liver cancer sample group was significantly higher compared with the normal group, while no significant difference was observed between primary liver cancer tissue samples and the adjacent non-cancerous liver tissue samples (Fig. 1 and Table I). As shown in Table II, PLSCR1 expression was observed in almost all primary liver cancer patients $(88.64 \%, 39 / 44$ patients), whereas the positive rate of PLSCR1 expression in the normal group was only $10 \%$. The positive rate of PLSCR1 expression increased with the increase of tumour size, although the difference was not statistically significant $(83.33 \%$ vs. $90.62 \%)$. The expression of PLSCR1 in primary liver cancer was correlated with the hepatitis B virus (HBV) infection status. Furthermore, it was identified that the positive rate of PLSCR1 expression in TNM stage III/IV samples was significantly higher compared with that in stage I/II samples, suggesting that the expression level of PLSCR1 is correlated with clinical stage. However, no correlations were identified between PLSCR1 expression and other clinicopathological characteristics, including patient sex, tumour size, tumour differentiation, $\alpha$ fetoprotein level and lymph node metastasis status. 
A

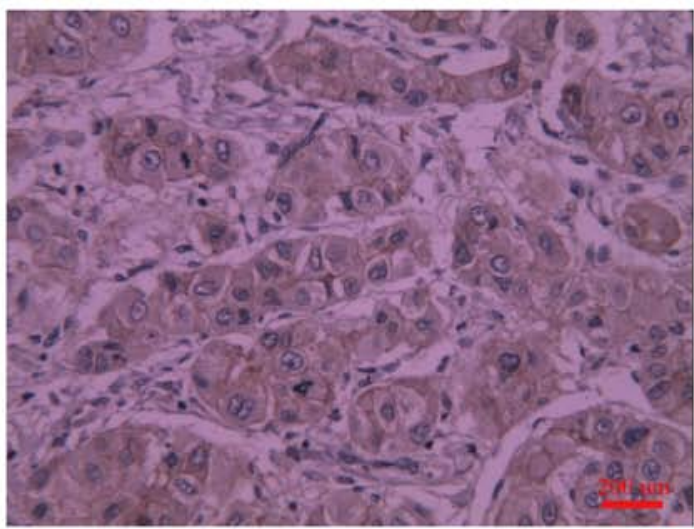

B

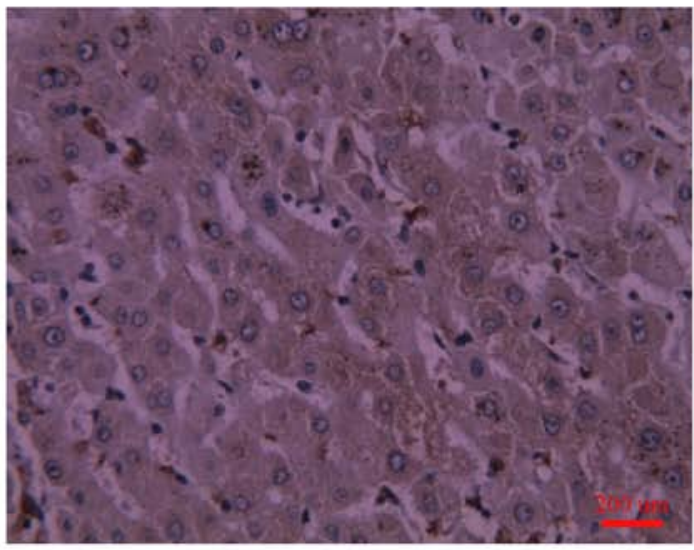

C

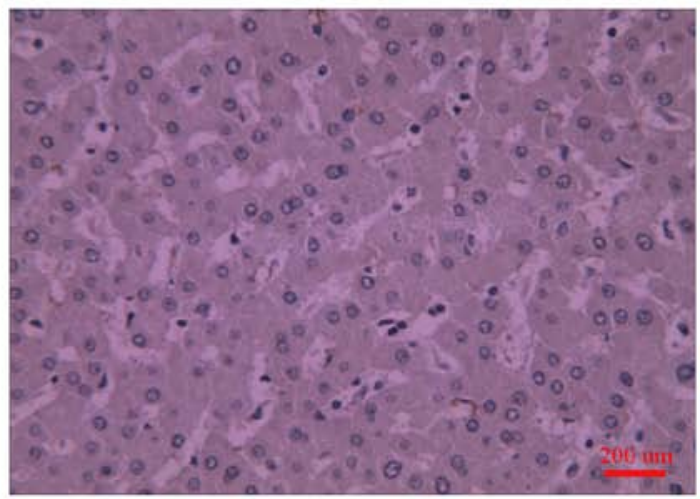

Figure 1. Expression and localization of PSCR1 in liver tissue samples analysed by immunohistochemical staining (magnification, $x 400$ ). Upregulated expression of PLSCR1 in (A) primary liver cancer and (B) adjacent non-cancerous liver tissue samples. (C) Negative staining for PLSCR1 in normal liver tissue samples. PSCR1, phospholipid scramblase 1.

PLSCR1 expression in transfected cancer cells. Following transfection with sh-PLSCR1, Lv-PLSCR1 or the corresponding negative control, the expression levels of PLSCR1 in the various groups was assessed using RT-qPCR and western blotting. The sh-PLSCR1 cell lines expressed the lowest levels of PLSCR1 at both the mRNA and protein levels, while significantly increased expression levels of PLSCR1 was observed in the Lv-PLSCR1 group compared with the other three groups (Fig. 2).

Effects of PLSCR1 expression on cell proliferation, adhesion, migration and invasion. Following transfection, PLSCR1 was overexpressed in the Lv-PLSCR1 cells, and the expression levels of PLSCR1 were reduced in the sh-PLSCR1 cells
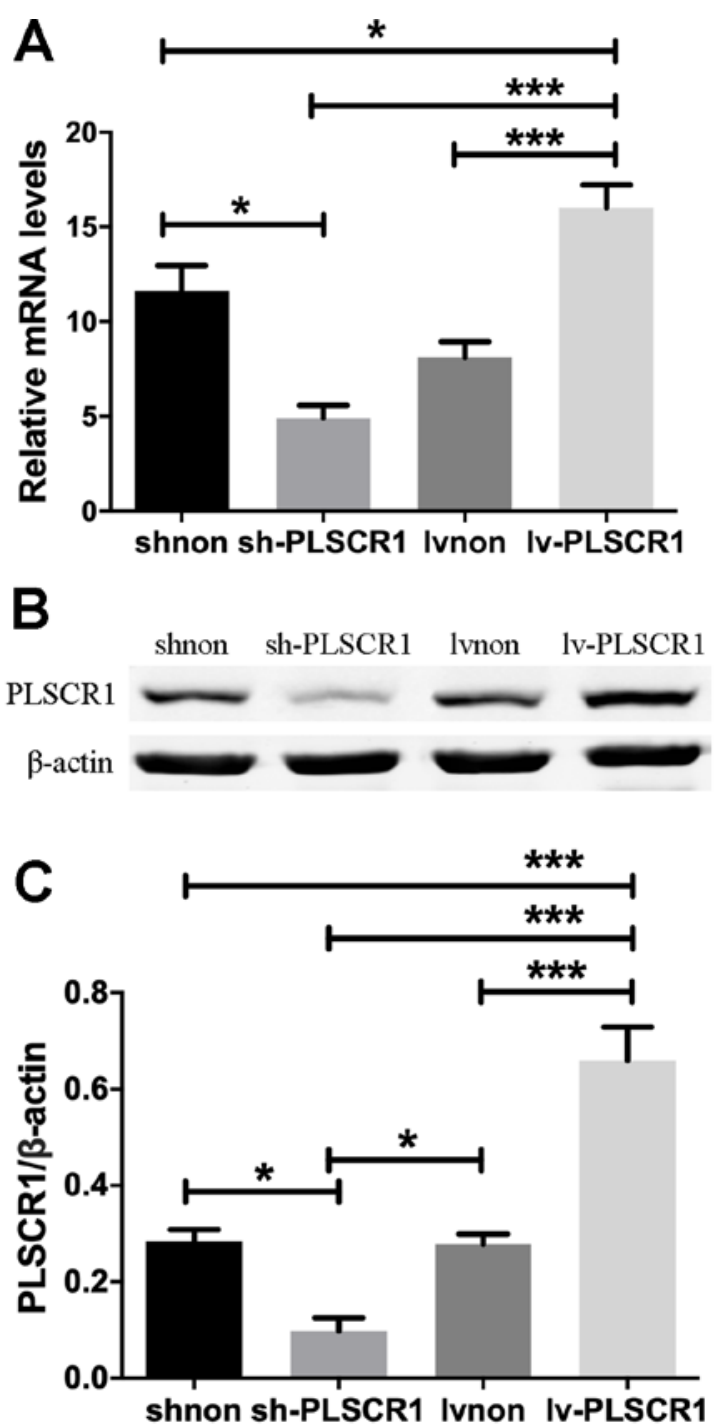

Figure 2. PLSCR1 expression in transfected cancer cells (A) PLSCR1 mRNA expression determined by reverse transcription-quantitative PCR. The results were normalized with $\beta$-actin used as the control. (B) Western blotting analyses and (C) quantification for PLSCR1. Quantitative analysis of the bands was performed using LabWorks analysis software. Data are presented as the mean \pm SD. ${ }^{*} \mathrm{P}<0.05,{ }^{* * *} \mathrm{P}<0.001$. lv, lentivirus; PSCR1, phospholipid scramblase 1; sh, short hairpin RNA.

as aforementioned. To investigate the effects of PLSCR1 on the biological behaviour of HepG2 cells, a series of assays including CCK8, adhesion and Transwell migration and invasion assays were performed. As shown in Fig. $3 \mathrm{~A}$ and $\mathrm{B}$, the CCK8 assays indicated that compared with the Lv-PLSCR1 cells, the sh-PLSCR1 cells exhibited significantly inhibited proliferation at 24 and $48 \mathrm{~h}$. Representative micrographs of Transwell filters are shown in Fig. 3C. Transwell migration and wound heal assays demonstrated that PLSCR1 overexpression resulted in an increased number of migrating Lv-PLSCR1 cells and promoted the migration of cancer cells (Fig. 3C-E). In addition, the number of invasive HepG2 cells was significantly decreased in the sh-PLSCR1 group compared with the Lv-PLSCR1 group (Fig. 4A and B). Ln and Fn adhesion assays were performed to assess HepG2 cell adhesion following transfection. These assays demonstrated that HepG2 cell adhesion was inhibited in the sh-PLSCR1 group 
A

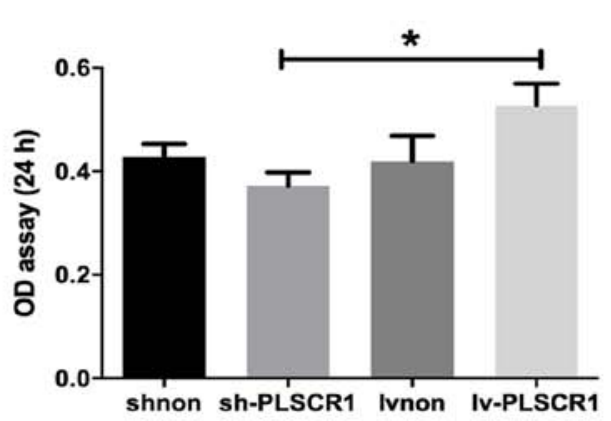

C

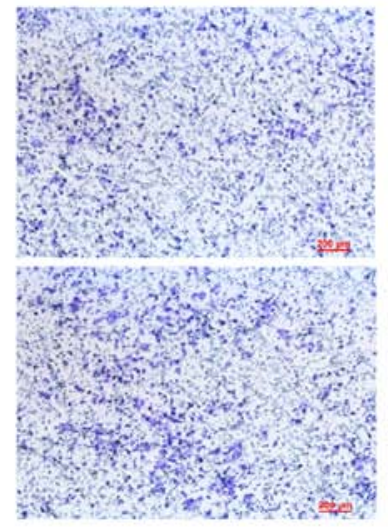

$\mathbf{E}$

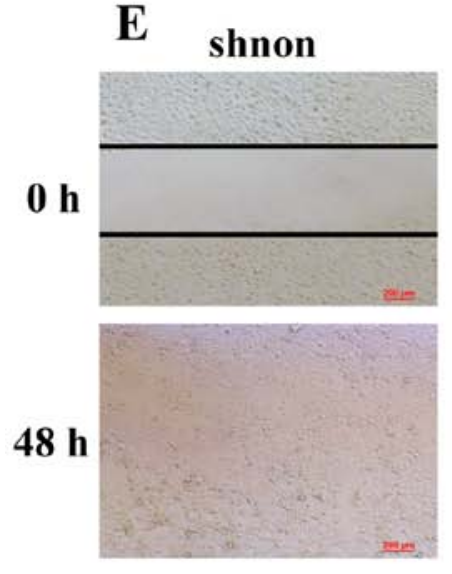

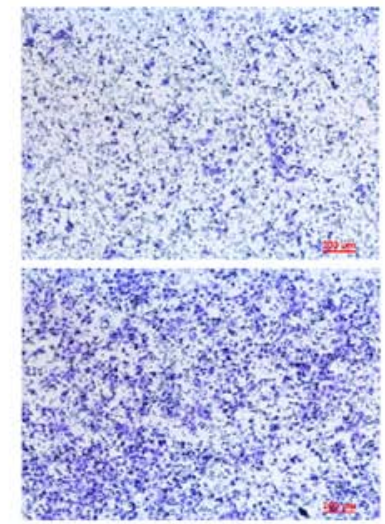

sh-PLSCR1
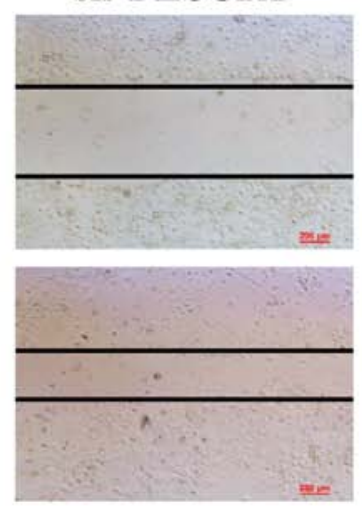

B

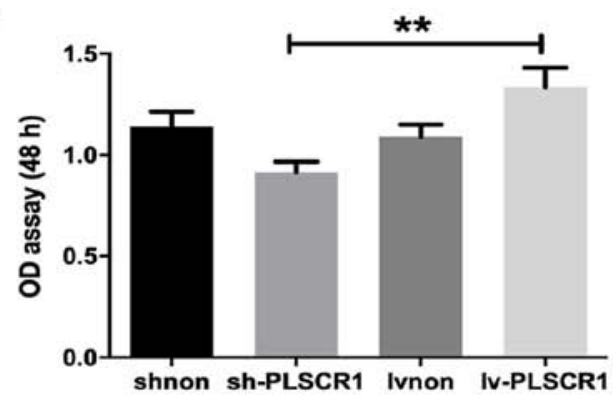

D

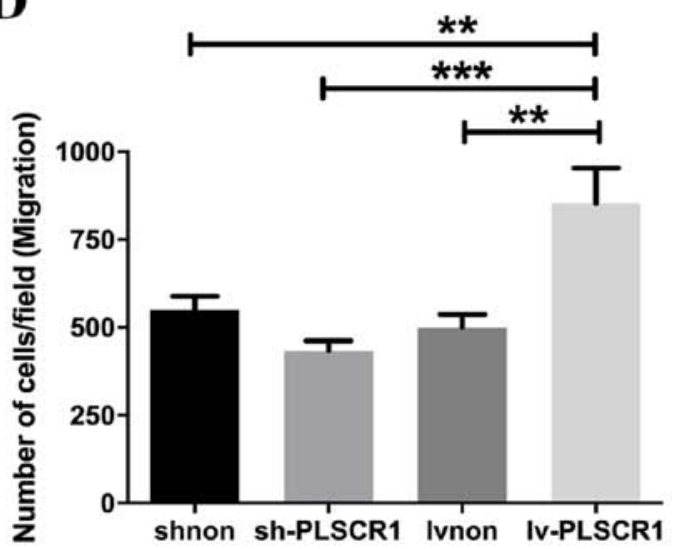

lvnon

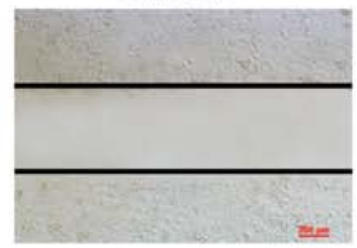

IV-PLSCR1
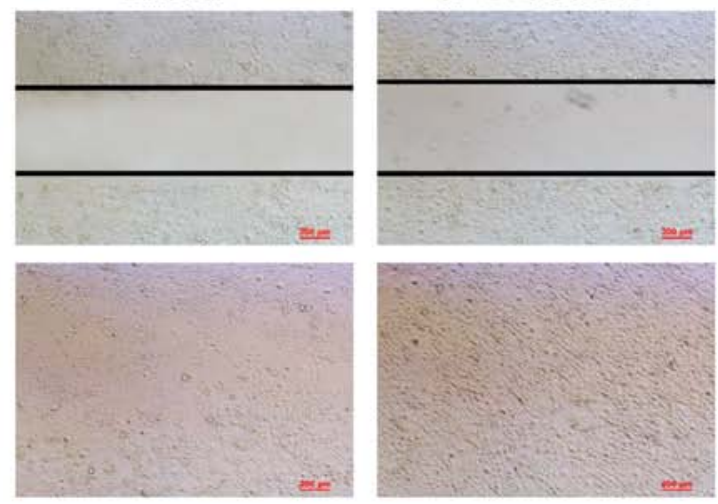

Figure 3. Lentivirus-mediated overexpression of PLSCR1 promotes HepG2 cell proliferation and migration in vitro. Cell proliferation determined by CCK8 assays at (A) 24 and (B) 48 h. (C) Representative images of Transwell migration assays of cells transfected with (a) shnon, (b) sh-PLSCR1, (c) Lvnon or (d) Lv-PLSCR1. (D) Quantification of the transwell migration assays. (E) Wound heal assay. Data are presented as the mean $\pm \mathrm{SD}$. ${ }^{*} \mathrm{P}<0.05,{ }^{* * *} \mathrm{P}<0.01,{ }^{* * * *} \mathrm{P}<0.001$. PSCR1, phospholipid scramblase 1; sh, short hairpin RNA; lv, lentivirus.

at $48 \mathrm{~h}$ (Fig. 4C and D). Collectively, the data suggested that PLSCR1 upregulation may promote HepG2 cell proliferation, migration and invasion and that PLSCR1 downregulation can inhibit these effects.

Suppression of PLSCRI expression blocks the EGFR signalling pathway. As shown in Fig. 5, expression of EGFR was decreased in cancer cells following PLSCR1 inhibition but increased in the PLSCR1-overexpression cells.

\section{Discussion}

Primary liver cancer is considered a serious health problem of global importance (19). The majority of patients are asymptomatic in the early stage but are often diagnosed in advanced stages, which are resistant to therapies $(4,20)$. Surgery is an optimal approach for primary liver cancer treatment, but the majority of patients may not be able to benefit from surgery due to a high rate of recurrence (21). Therefore, early diagnosis and the identification of new therapeutic targets will benefit patients with primary liver cancer.

As aforementioned, increasing evidence suggests that PLSCR1 serves important roles in cell proliferation, differentiation, apoptosis and cancer pathogenesis and progression (17). Studies have demonstrated that PLSCR1 overexpression is associated with the differentiation of human myeloid leukaemia cells into granulocytes and inhibits the growth of ovarian cancer cells $(9,22)$. Other studies have revealed that suppression of PLSCR1 inhibits the growth and metastasis of colorectal cancer $(16,23)$. Nevertheless, the roles of PLSCR1 
A

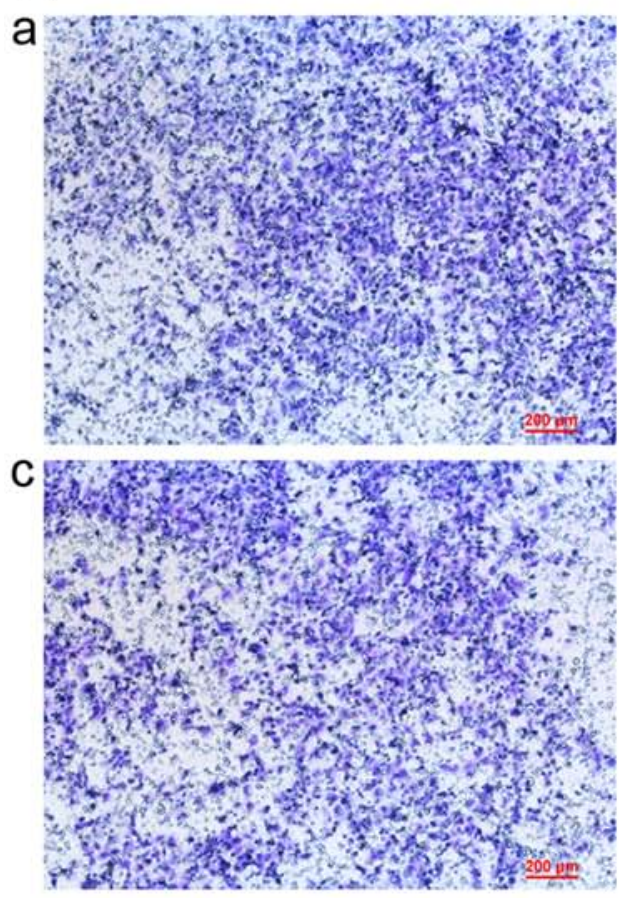

$\mathrm{B}$
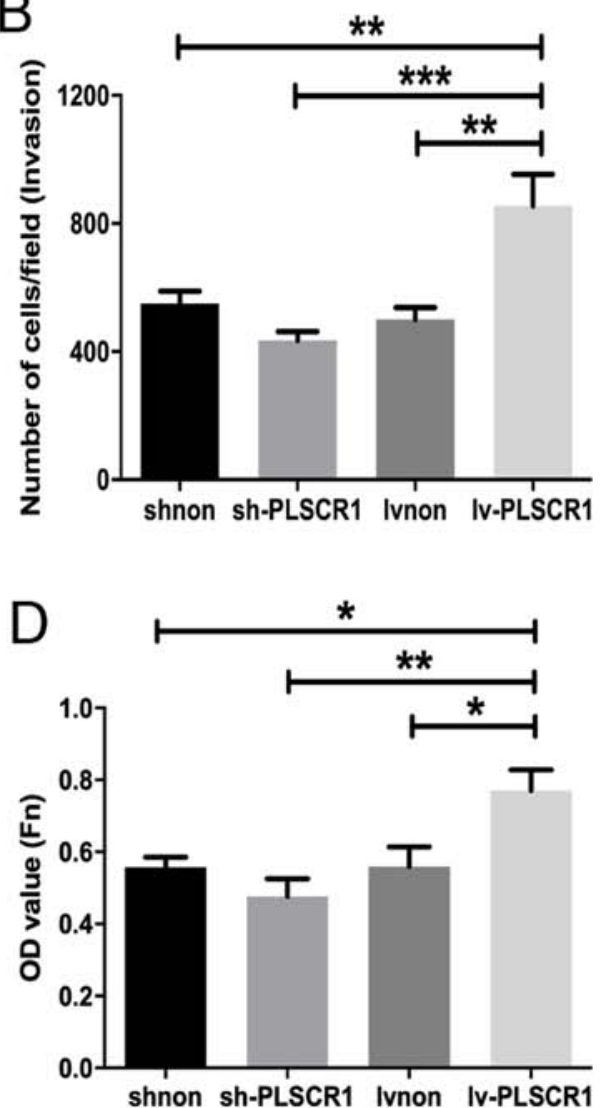
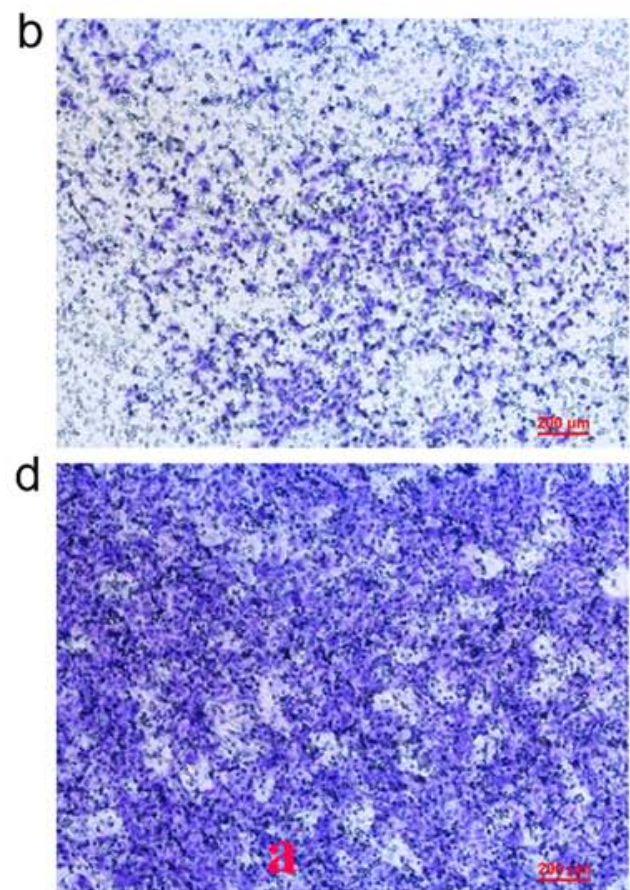

C

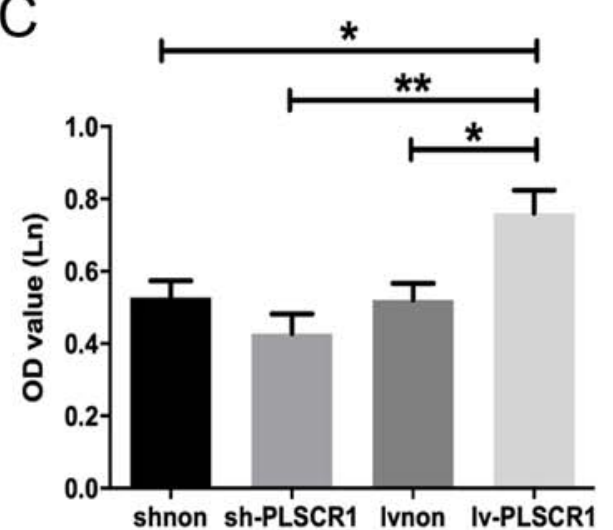

Figure 4. Lentivirus-mediated overexpression of PLSCR1 promotes HepG2 cell invasion and adhesion in vitro. (A) Representative images of cell invasion determined by Transwell invasion assays of cells transfected with (a) shnon, (b) sh-PLSCR1, (c) Lvnon or (d) Lv-PLSCR1. (B) Quantification of the Transwell invasion assays. (C) Ln and (D) Fn adhesion assays. Data are presented as the mean \pm SD. ${ }^{*} \mathrm{P}<0.05,{ }^{* *} \mathrm{P}<0.01,{ }^{* * *} \mathrm{P}<0.001$. OD, optical density; PSCR1, phospholipid scramblase 1; sh, short hairpin RNA; lv, lentivirus; Ln, laminin; Fn, fibronectin.

in the pathogenesis and progression of primary liver cancer remain to be elucidated.

In the present study, there was a strong correlation between PLSCR1 expression and tumor stage. Univariate analysis with the Cox regression model demonstrates that increased PLSCR 1 expression indicates a poor prognosis for patients with colorectal cancer $(16,17)$. Based on these results and previous studies, it was hypothesized that PLSCR1 may serve 
A

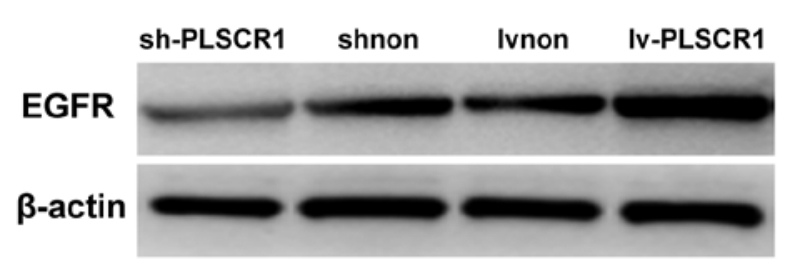

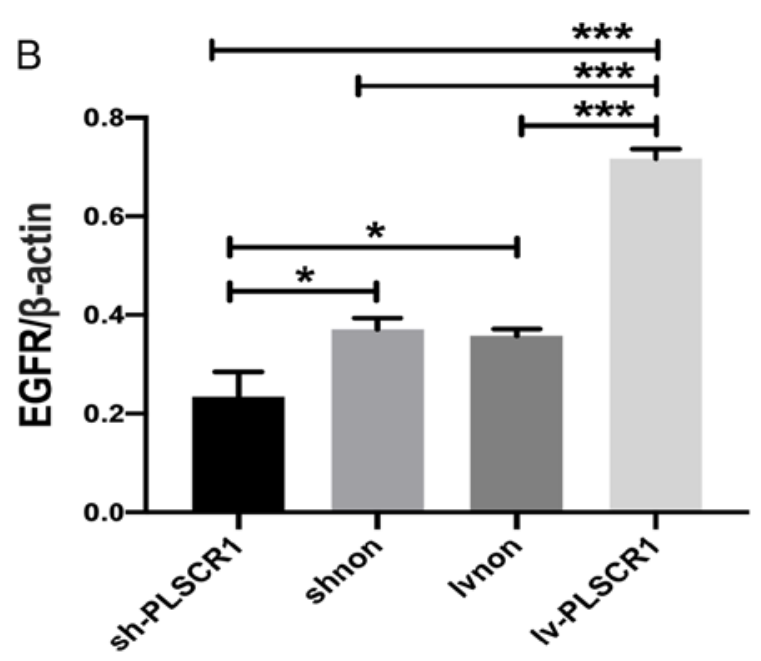

Figure 5. Western blotting analyses for EGFR. (A) Representative images of western blots. (B) Quantitative analysis of the bands was performed using LabWorks analysis software. Data are presented as the mean $\pm \mathrm{SD} .{ }^{*} \mathrm{P}<0.05,{ }^{* * *} \mathrm{P}<0.001$. EGFR, epidermal growth factor receptor; sh, short hairpin RNA; lv, lentivirus; PSCR1, phospholipid scramblase 1.

a vital role behind the tumorigenesis of primary liver cancer. The present study also demonstrated that the expression of PLSCR1 in primary liver cancer was associated with the HBs-Ag status. Yuan et al (24) reported an important role for PLSCR1 in the host defence against HBV infection and the development of primary liver cancer in patients with HBV, which are consistent with the findings of the present study.

To evaluate the biological function of PLSCR1, siRNA and lentiviral technologies were used to regulate the expression of PLSCR1 and the changes in expression were verified by RT-qPCR and western blotting. The results demonstrated that proliferation, adhesion, migration and invasion were blocked following the suppression of PLSCR1 expression and promoted following upregulation of PLSCR1 expression, suggesting that PLSCR 1 could be a potential therapeutic target for hepatocellular carcinoma. Previous studies have indicated that the EGFR signalling pathway is inhibited in cells with knocked down PLSCR1 expression $(9,22)$. Stimulation of EGFR-expressing cells via EGF causes PLSCR1 to interact with activated EGFR and SHC Adaptor Protein 1 (Shc), which results in the tyrosine phosphorylation of PLSCR1 (25). PLSCR1 is a substrate of multiple kinases, involved in cell proliferation, differentiation or apoptosis including tyrosine-protein kinase ABL1, proto-oncogene tyrosine-protein kinase Src (Src) and protein kinase (14,26-28). In EGF-treated cells, activated Src kinase causes the tyrosine phosphorylation of PLSCR1, which is required for the interaction of PLSCR1 with Shc in response to EGF stimulation (14). In the present study, the production of EGFR was decreased following suppression of PLSCR1 expression and increased following upregulation of PLSCR1. Therefore, suppression of PLSCR1 expression may block the EGFR signalling pathway and inhibit the proliferation, adhesion, migration and invasion of HepG2 cells. However, a limitation of the present study is that the downstream proteins inside the EGFR signal penetration pathway were not measured, which would further support the reduction in EGFR signalling. Lack of in vivo experiments is another limitation of the present study and which should be further investigated.
In summary, the present study indicated that PLSCR1 was highly expressed in primary liver cancer and associated with the tumour stage. Downregulating the expression of PLSCR1 significantly inhibited the proliferation, adhesion, migration and invasion of cancer cells. PLSCR1 overexpression may serve important roles in tumorigenesis and tumour progression, and suppressing PLSCR1 expression may be beneficial for the treatment of primary liver cancer. However, further research needs to be conducted to explore the potential molecular mechanisms of the signalling pathways involved.

\section{Acknowledgements}

Not applicable.

\section{Funding}

This work was supported by i) Gastroenterology of key disciplines from Wuxi (grant no. DXK002), ii) Academician Workstation of No. 2 People's Hospital of Wuxi City (grant no. CYR1705), iii) Wuxi Medical Key Talent Project (grant no. ZDRC029), iv) Wuxi Sci-Tech Development Fund (grant no. CSE31N1603), v) Technical achievements and appropriate technology promotion project of Wuxi Municipal Commission of Health and Family Planning (grant no. T201603), vi) Wuxi municipal commission of health and family planning project (grant no. MS201720), vii) Wuxi municipal commission of health and family planning project (grant no. MS201814), viii) Wujin district Changzhou Sci-Tech Development Fund (grant no. WS201811), ix) Jiangsu commission of health project (grant no. LGY2018021) and x) Jiangsu University clinical medical science and technology development fund (grant no. JLY20180090).

\section{Availability of data and materials}

The datasets used and analysed during the current study are available from the corresponding author on reasonable request. 


\section{Authors' contributions}

LG, YZ and QX designed the study, performed the experiments and analysed the data statistically. LG wrote the manuscript. $\mathrm{JH}, \mathrm{PH}$ and GW performed data collection. LZ and JL made substantial contributions to the conception of this study. JN and HT conducted data processing and statistical analyses, and made critical revisions. All authors read and approved the final manuscript.

\section{Ethics approval and consent to participate}

The study was approved by the Ethics Committee of Wuxi No. 2 People's Hospital, and written informed consent was obtained from all the patients prior to surgery.

\section{Patient consent for publication}

Not applicable.

\section{Competing interests}

The authors declare that they have no competing interests.

\section{References}

1. Grandhi MS, Kim AK, Ronnekleiv-Kelly SM, Kamel IR, Ghasebeh MA and Pawlik TM: Hepatocellular carcinoma: From diagnosis to treatment. Surg Oncol 25: 74-85, 2016.

2. Zhu Y, Tang H, Zhang L, Gong L, Wu G, Ni J and Tang X Suppression of miR-21-3p enhances TRAIL-mediated apoptosis in liver cancer stem cells by suppressing PI3K/Akt/Bad cascade via regulating PTEN. Cancer Manag Res 11: 955-968, 2019.

3. Balogh J, Victor D III, Asham EH, Burroughs SG, Boktour M, Saharia A, Li X, Ghobrial RM and Monsour HP Jr: Hepatocellular carcinoma: A review. J Hepatocell Carcinoma 3: 41-53, 2016.

4. Gomes MA, Priolli DG, Tralhao JG and Botelho MF: Hepatocellular carcinoma: Epidemiology, biology, diagnosis, and therapies. Rev Assoc Med Bras (1992) 59: 514-524, 2013 (In English, Portuguese).

5. Cidon EU: Systemic treatment of hepatocellular carcinoma: Past, present and future. World J Hepatol 9: 797, 2017.

6. Wiedmer T, Zhao J, Nanjundan M and Sims PJ: Palmitoylation of phospholipid scramblase 1 controls its distribution between nucleus and plasma membrane. Biochemistry 42: 1227-1233, 2003.

7. Zhou Q, Zhao J, Wiedmer T and Sims PJ: Normal hemostasis but defective hematopoietic response to growth factors in mice deficient in phospholipid scramblase 1. Blood 99: 4030-4038, 2002.

8. Huang Y, Zhao Q, Zhou CX, Gu ZM, Li D, Xu HZ, Wiedmer T, Sims PJ, Zhao KW and Chen GQ: Antileukemic roles of human phospholipid scramblase 1 gene, evidence from inducible PLSCR1-expressing leukemic cells. Oncogene 25: 6618-6627, 2006.

9. Silverman RH, Halloum A, Zhou A, Dong B, Al-Zoghaibi F, Kushner D, Zhou Q, Zhao J, Wiedmer T and Sims PJ: Suppression of ovarian carcinoma cell growth in vivo by the interferon-inducible plasma membrane protein, phospholipid scramblase 1. Cancer Res 62: 397-402, 2002.

10. Francis VG, Padmanabhan P and Gummadi SN: Snail interacts with hPLSCR1 promoter and down regulates its expression in IMR-32. Biochem Biophys Res Commun 450: 172-177, 2014.

11. Vinnakota JM and Gummadi SN: Two c-Myc binding sites are crucial in upregulating the expression of human phospholipid scramblase 1 gene. Biochem Biophys Res Commun 469: 412-417, 2016.
12. Zhou Q, Ben-Efraim I, Bigcas J, Junqueira D, Wiedmer T and Sims PJ: Phospholipid scramblase 1 binds to the promoter region of the inositol 1,4,5-triphosphate receptor type 1 gene to enhance its expression. J Biol Chem 280: 35062-35068, 2005.

13. Sahu SK, Gummadi SN, Manoj $\mathrm{N}$ and Aradhyam GK: Phospholipid scramblases: An overview. Arch Biochem Biophys 462: 103-114, 2007.

14. Nanjundan M, Sun J, Zhao J, Zhou Q, Sims PJ and Wiedmer T: Plasma membrane phospholipid scramblase 1 promotes EGF-dependent activation of c-Src through the epidermal growth factor receptor. J Biol Chem 278: 37413-37418, 2003.

15. Choi HJ, Lui A, Ogony J, Jan R, Sims PJ and Lewis-Wambi J: Targeting interferon response genes sensitizes aromatase inhibitor resistant breast cancer cells to estrogen-induced cell death. Breast Cancer Res 17: 6, 2015.

16. Cui W, Li SY, Du JF, Zhu ZM and An P: Silencing phospholipid scramblase 1 expression by RNA interference in colorectal cancer and metastatic liver cancer. Hepatob Pancreat Dis 11: 393-400, 2012.

17. Kuo YB, Chan CC, Chang CA, Fan CW, Hung RP, Hung YS, Chen KT, Yu JS, Chang YS and Chan EC: Identification of phospholipid scramblase 1 as a biomarker and determination of its prognostic value for colorectal cancer. Mol Med 17: 41-47, 2011.

18. Livak KJ and Schmittgen TD: Analysis of relative gene expression data using real-time quantitative PCR and the 2(-Delta Delta C(T)) method. Methods 25: 402-408, 2001.

19. Singal AG and El-Serag HB: Hepatocellular carcinoma from epidemiology to prevention: Translating knowledge into practice. Clin Gastroenterol Hepatol 13: 2140-2151, 2015.

20. Finn RS: Emerging targeted strategies in advanced hepatocellular carcinoma. Semin Liver Dis 33 (Suppl 1): S11-S19, 2013.

21. Brito AF, Abrantes AM, Tralhão JG and Botelho MF: Targeting hepatocellular carcinoma: What did we discover so far? Oncol Rev 10: 302, 2016

22. Nakamaki T, Okabe-Kado J, Yamamoto-Yamaguchi Y, Hino Ki, Tomoyasu S, Honma Y and Kasukabe T: Role of MmTRA1b/phospholipid scramblasel gene expression in the induction of differentiation of human myeloid leukemia cells into granulocytes. Exp Hematol 30: 421-429, 2002.

23. Fan CW, Chen CY, Chen KT, Shen CR, Kuo YB, Chen YS, Chou YP, Wei WS and Chan EC: Blockade of phospholipid scramblase 1 with its $\mathrm{N}$-terminal domain antibody reduces tumorigenesis of colorectal carcinomas in vitro and in vivo. J Transl Med 10: 254, 2012.

24. Yuan Y, Tian C, Gong Q, Shang L, Zhang Y, Jin C, He F and Wang J: Interactome map reveals phospholipid scramblase 1 as a novel regulator of hepatitis B virus x protein. J Proteome Res 14: $154-163,2015$.

25. Yokoyama A, Yamashita $T$, Shiozawa E, Nagasawa A, Okabe-Kado J, Nakamaki T, Tomoyasu S, Kimura F, Motoyoshi K, Honma Y and Kasukabe T: MmTRA1b/phospholipid scramblase 1 gene expression is a new prognostic factor for acute myelogenous leukemia. Leuk Res 28: 149-157, 2004.

26. Sun J, Zhao J, Schwartz MA, Wang JY, Wiedmer T and Sims PJ: $\mathrm{c}$-Abl tyrosine kinase binds and phosphorylates phospholipid scramblase 1. J Biol Chem 276: 28984-28990, 2001.

27. Kowalczyk JE, Beręsewicz M, Gajkowska B and Zabłocka B: Association of protein kinase $\mathrm{C}$ delta and phospholipid scramblase 3 in hippocampal mitochondria correlates with neuronal vulnerability to brain ischemia. Neurochem Int 55: 157-163, 2009.

28. Zhao KW, Li X, Zhao Q, Huang Y, Li D, Peng ZG, Shen WZ, Zhao J, Zhou Q, Chen Z, et al: Protein kinase Cdelta mediates retinoic acid and phorbol myristate acetate-induced phospholipid scramblase 1 gene expression: Its role in leukemic cell differentiation. Blood 104: 3731-3738, 2004.

This work is licensed under a Creative Commons Attribution-NonCommercial-NoDerivatives 4.0 International (CC BY-NC-ND 4.0) License. 